\title{
Impact and Use of the Asymmetric Property in Bi-directional Cooperative Relaying under Asymmetric Traffic Conditions
}

\author{
Takaaki SAEKI $^{\dagger \text { a) }}$, Koji YAMAMOTO ${ }^{\dagger}$, Members, Hidekazu MURATA ${ }^{\dagger}$, Senior Member, \\ and Susumu YOSHIDA ${ }^{\dagger}$, Fellow
}

\begin{abstract}
SUMMARY Cooperative relaying (CR) is a promising technique to provide spatial diversity by combining multiple signals from source and relay stations. In the present paper, the impact and use of the asymmetric property in bi-directional CR under asymmetric traffic conditions are discussed assuming that $\mathrm{CR}$ involves one communication pair and one relay station in a time division duplex (TDD) system. The asymmetric property means that the average communication quality differs for each transmission direction because of the difference in signal power between the combined signals for each direction. First, numerical results show the asymmetric property of bi-directional CR. Next, in order to evaluate the impact of the asymmetric property, the optimal relay position and resource allocation are compared to those in simple multi-hop relaying, which does not have the asymmetric property. Numerical results show that, in order to maximize the overall quality of bi-directional communication, the optimal relay position in CR depends on the offered traffic ratio, which is defined as the traffic ratio of each transmission direction, while the offered traffic ratio does not affect the optimal relay position in multi-hop relaying. Finally, the asymmetric property is used to enhance the overall quality. Specifically, a high overall quality can be achieved by, for example, opportunistically switching to the transmission direction with higher quality. Under asymmetric traffic conditions, weighted proportionally fair scheduling (WPFS), which is proposed in the context of downlink scheduling in a cellular network, is applied to transmission direction switching. Numerical results reveal that WPFS provides a high overall quality and that the quality ratio is similar to the offered traffic ratio.

key words: cooperative relaying, asymmetric traffic, optimal relay position, opportunistic scheduling
\end{abstract}

\section{Introduction}

Cooperative relaying (CR) is a promising technique to provide spatial diversity by combining multiple signals from the source and relay stations. The performance of CR has been evaluated in terms of error rate and spectral efficiency in unidirectional communication [1]-[4]. However, in most practical communication systems, the destination also has data to send back to the source, e.g., control signaling or traffic in both directions. Thus, bi-directional or two-way communication via relay station has also been actively investigated [5]-[11].

Bi-directional CR with decode-and-forward protocol has an asymmetric property, i.e., the average communication quality differs for each transmission direction because of the difference in signal power between the combined signals for each direction, under the assumption that the same

Manuscript received October 29, 2009.

Manuscript revised April 22, 2010.

${ }^{\dagger}$ The authors are with the Graduate School of Informatics, Kyoto University, Kyoto-shi, 606-8501 Japan.

a)E-mail: contact-h22@ hanase.kuee.kyoto-u.ac.jp

DOI: 10.1587/transcom.E93.B.2126 relay stations are used for both transmission directions and all stations transmit at the same power. However, to the best of the authors' knowledge, the asymmetric property has not been considered in previous studies. Most previous research on bi-directional relaying has focused on network coding or superposition coding to reduce the number of transmissions and enhance aspects of performance such as the total throughput and error rate [5]-[8].

A number of studies have assumed symmetric traffic or have not assumed a specific traffic ratio. In [9], the authors proposed a regenerative strategy to improve the fairness, i.e., to maximize the minimum unidirectional transmission rate. In [5]-[8], only the total throughput enhancement is discussed; the throughput ratio for each direction is not considered. In [10], the authors derived the achievable rate regions of various bi-directional relaying protocols and determined their relative performance under different relay geometries, assuming an asymmetric traffic ratio. However, they focused primarily on symmetric traffic and did not specifically discuss the impact of asymmetric traffic when maximizing the total throughput. In [11], the authors investigated the influence of the traffic pattern on the performance of network coding in terms of the total throughput and concluded that the network coding schemes do not provide significant rate gain when the two-way relaying system has a very asymmetric traffic pattern. However, they fixed the relay position and discussed the maximum total throughput with respect to only the traffic pattern.

In order to maximize the total throughput under asymmetric traffic conditions, the impact of the asymmetric property on the optimal relay position must be clarified. This is because the asymmetric property may cause the optimal relay position to change depending on the offered traffic ratio, which is defined as the traffic ratio of each transmission direction, resulting in enhanced total throughput. Note that in multi-hop relaying there is no need to evaluate the impact of the asymmetric property on the optimal position, because multi-hop relaying does not have the asymmetric property.

Therefore, in the present paper, the asymmetric property is considered, and the impact of the asymmetric property on the optimal relay position under asymmetric conditions is evaluated. Since our primary motivation is not to propose protocols, but rather to evaluate the property and enhance the total throughput, we compare a simple CR with a simple multi-hop relaying, in which four phases are required to communicate in both directions. 
Furthermore, in order to enhance the total throughput of bi-directional CR by exploiting the asymmetric property, an opportunistic transmission direction switching scheme is proposed. As stated above, the average communication quality in bi-directional CR differs for each transmission direction and the instantaneous communication quality also differs for each direction. Thus, opportunistic switching, in which the end stations switch to the direction with higher quality, can enhance the overall quality of bi-directional communication.

The remainder of the present paper is organized as follows. In Sect. 2, the system model is described. In Sect. 3, we show the asymmetric property of bi-directional CR. In Sect. 4, we define the resource allocation problem under asymmetric traffic conditions and evaluate the impact of the asymmetric property on resource allocation, as compared to that of two-hop relaying. In Sect. 5, we evaluate the average throughput of the opportunistic transmission direction switching schemes exploiting the asymmetric property. Section 6 summarizes the findings of the present study.

\section{System Model}

The system model considered in the present paper is shown in Fig. 1. In this model, stations $1\left(\mathrm{~S}_{1}\right)$ and $2\left(\mathrm{~S}_{2}\right)$ communicate with each other using one relay station (R) in the TDD mode. The relay station is assumed to decode and forward the received signal using two temporal phases, the durations of which are equal, and all stations are assumed to transmit at the same power. In Sect. 4, we assume CR and two-hop relaying and address the difference in optimal relay position between them. In Sect. 5, we address the opportunistic transmission direction switching schemes for CR and evaluate their average throughputs. In the following, for the sake of simplicity, we explain the system model in the case of communication only from $S_{1}$ to $S_{2}$, as shown in Fig. 2.

Cooperative relaying This scheme is proposed in [2], [12]. In phase 1 , both $R$ and $S_{2}$ receive the signal from $S_{1}$. In phase 2, only $R$ transmits to $S_{2}$.

Two-hop relaying In phase 1, only $\mathrm{R}$ receives the signal from $S_{1}$. In phase 2, R transmits to $S_{2}$.

\subsection{Cooperative Relaying}

We introduce instantaneous end-to-end throughput as a measure of quality. The instantaneous throughput of the CR from $\mathrm{S}_{1}$ to $\mathrm{S}_{2}$ at time $t$, denoted as $f_{\mathrm{S}_{1} \rightarrow \mathrm{S}_{2}}^{\mathrm{CR}}(t)$, is assumed to be expressed as

$$
f_{\mathrm{S}_{1} \rightarrow \mathrm{S}_{2}}^{\mathrm{CR}}(t)=\frac{1}{2} \min \left\{f_{\mathrm{S}_{1} \rightarrow \mathrm{S}_{2}}^{1, \mathrm{CR}}(t), f_{\mathrm{S}_{1} \rightarrow \mathrm{S}_{2}}^{2, \mathrm{CR}}(t)\right\}
$$

where

$$
\begin{aligned}
& f_{\mathrm{S}_{1} \rightarrow \mathrm{S}_{2}}^{1, \mathrm{CR}}(t)=\log _{2}\left(1+\left|h_{\mathrm{S}_{1} \mathrm{R}}\right|^{2} \overline{\gamma_{\mathrm{S}_{1} \mathrm{R}}}\right) \\
& f_{\mathrm{S}_{1} \rightarrow \mathrm{S}_{2}}^{2, \mathrm{CR}}(t)=\log _{2}\left(1+\left|h_{\mathrm{S}_{1} \mathrm{~S}_{2}}\right|^{2} \bar{\gamma}+\left|h_{\mathrm{RS}_{2}}\right|^{2} \overline{{\mathrm{RS}_{2}}_{2}}\right),
\end{aligned}
$$

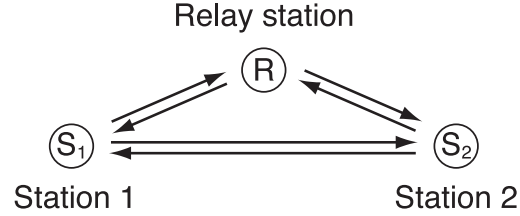

Fig. 1 Model of the bi-directional cooperative relay channel.

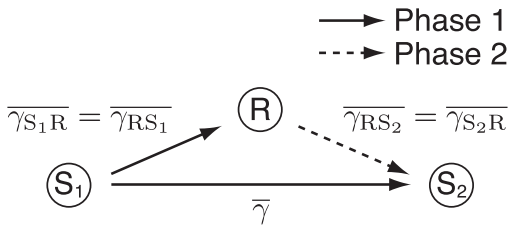

(a) Cooperative relaying scheme.

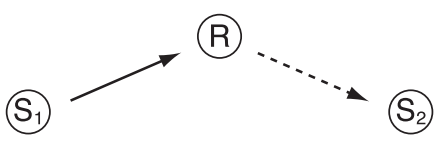

(b) Two-hop relaying scheme.

Fig. 2 Relaying schemes considered in the present paper, where $\bar{\gamma}$ denotes the average received SNR through the $\mathrm{S}_{1}-\mathrm{S}_{2}$ link, $\overline{\gamma_{X Y}}$ denotes the average received SNR at station $Y$ through the station $X$-station $Y$ link.

as in [1], [4], which is achieved through maximal ratio combining of the signals from $S_{1}$ and R. Here, $\bar{\gamma}$ denotes the average end-to-end signal-to-noise power ratio (SNR), i.e., the average received SNR through the $\mathrm{S}_{1}-\mathrm{S}_{2}$ link, $\overline{\gamma_{X Y}}$ denotes the average received SNR at station $Y$ through the station $X$-station $Y$ link, $h_{X Y}$ denotes a complex unit-power channel gain through the station $X$-station $Y$ link at time $t$, and the factor $1 / 2$ represents two phases required for this scheme [2]. $\overline{\gamma_{X Y}}$ can be expressed as $\overline{\gamma_{X Y}}=\left(d_{X Y} / d_{\mathrm{S}_{1} \mathrm{~S}_{2}}\right)^{-\alpha} \bar{\gamma}$ with the distance between stations $X$ and $Y d_{X Y}$. We assume a flat Rayleigh block-fading channel, where $h_{X Y}$ is assumed to be i.i.d. $\mathcal{C N}(0,1)$ and not to change within two phases. Note that $h_{X Y} \neq h_{Y X}$ but $\mathrm{E}\left[\left|h_{X Y}\right|\right]=\mathrm{E}\left[\left|h_{Y X}\right|\right]$, where $\mathrm{E}[\cdot]$ denotes the expectation operator.

Similarly, the instantaneous throughput of the CR from $\mathrm{S}_{2}$ to $\mathrm{S}_{1}$ at time $t$, denoted as $f_{\mathrm{S}_{2} \rightarrow \mathrm{S}_{1}}^{\mathrm{CR}}(t)$, can be expressed as

$$
f_{\mathrm{S}_{2} \rightarrow \mathrm{S}_{1}}^{\mathrm{CR}}(t)=\frac{1}{2} \min \left\{f_{\mathrm{S}_{2} \rightarrow \mathrm{S}_{1}}^{1, \mathrm{CR}}(t), f_{\mathrm{S}_{2} \rightarrow \mathrm{S}_{1}}^{2, \mathrm{CR}}(t)\right\}
$$

where

$$
\begin{aligned}
& f_{\mathrm{S}_{2} \rightarrow \mathrm{S}_{1}}^{1, \mathrm{CR}}(t)=\log _{2}\left(1+\left|h_{\mathrm{S}_{2} \mathrm{R}}\right|^{2} \overline{\gamma_{\mathrm{S}_{2} \mathrm{R}}}\right) \\
& f_{\mathrm{S}_{2} \rightarrow \mathrm{S}_{1}}^{2, \mathrm{CR}}(t)=\log _{2}\left(1+\left|h_{\mathrm{S}_{1} \mathrm{~S}_{2}}\right|^{2} \bar{\gamma}+\left|h_{\mathrm{RS}_{1}}\right|^{2} \overline{{\mathrm{RS}_{1}}_{1}}\right) .
\end{aligned}
$$

\subsection{Two-Hop Relaying}

The instantaneous throughput of two-hop relaying at time $t$, denoted as $f^{2-\text { hop }}(t)$, can be expressed as

$$
\begin{aligned}
f_{\mathrm{S}_{1} \rightarrow \mathrm{S}_{2}}^{2-\text { hop }}(t) & =f_{\mathrm{S}_{2} \rightarrow \mathrm{S}_{1}}^{2-\text { hop }}(t) \\
& =\frac{1}{2} \min \left\{\log _{2}\left(1+\left|h_{\mathrm{S}_{1} \mathrm{R}}\right|^{2} \overline{{\mathrm{S}_{1} \mathrm{R}}_{1}}\right),\right.
\end{aligned}
$$




$$
\left.\log _{2}\left(1+\left|h_{\mathrm{RS}_{2}}\right|^{2} \overline{\gamma_{\mathrm{RS}_{2}}}\right)\right\} \text {. }
$$

\section{Asymmetric Property}

In this section, we show the asymmetric property of CR. This property is caused by the difference in signal power between the combined signals for each direction. For example, as shown in Fig. 2 (a), when $S_{1}$ transmits the signal to $\mathrm{S}_{2}, \mathrm{~S}_{2}$ combines the signals from $\mathrm{S}_{1}$ and $\mathrm{R}$. In the same way, when $S_{2}$ transmits the signal to $S_{1}, S_{1}$ combines the signals from $\mathrm{S}_{2}$ and $\mathrm{R}$.

Table 1 summarizes the parameters used in the evaluation. We assume path loss with path loss exponent $\alpha$. For the sake of simplicity, the effect of shadowing is not considered. Let $\left(x_{\mathrm{R}}, y_{\mathrm{R}}\right)$ denote the position of $\mathrm{R}$ when assuming the position of $S_{1}$ and $S_{2}$ are $(0,0)$ and $(1,0)$. First, $R$ is assumed to be located on a straight-line segment from $S_{1}$ to $S_{2}$. The average end-to-end SNR is set to $-10 \mathrm{~dB}$, where CR achieves a higher average throughput than single-hop transmission.

Figure 3(a) shows the average throughput for each direction. The horizontal axis shows the distance of $\mathrm{S}_{1}-\mathrm{R}$ normalized by that of $S_{1}-S_{2}$. The average throughput of CR differs for each direction when the relay station is not located halfway between end stations, while the average throughput of two-hop relaying does not differ. We also observe that the highest throughput of one direction is achieved when the relay station is located closer to the source than the midpoint. There are two reasons for this: 1) the combining gain of received signals at the destination and 2) the throughput constraint of the source-relay station link to decode the received signal at the relay station. Even when $\mathrm{R}$ is not on the straight line segment from $S_{1}$ to $S_{2}$, i.e., $y_{R} \neq 0$, we can also confirm that the average throughput of CR differs for each direction when $x_{\mathrm{R}} \neq 0.5$ as shown in Fig. 3 (b).

\section{Optimal Relay Position}

In this section, we evaluate the impact of the asymmetric property on the optimal relay position and resource allocation. Under asymmetric traffic conditions, resources should be allocated to maximize the overall quality of bi-directional communication under the constraint to achieve a given traffic ratio. This maximization is equivalent to a model in real systems, where each end station is required to send a different amount of data as fast as possible.

In single-hop transmission in TDD system, only time is commonly allocated to achieve the given ratio. In contrast, in relaying schemes, not only time allocation but also

Table 1 Parameters used in the present evaluation.

\begin{tabular}{c|c}
\hline Parameters & Values \\
\hline Path loss exponent $\alpha$ & 2 \\
Channel model & Rayleigh fading \\
Area environment & Noise-limited \\
Average end-to-end SNR $\bar{\gamma}$ & $-10 \mathrm{~dB}$ \\
\hline
\end{tabular}

the relay position should be optimized. Therefore, we assume these two parameters, namely, the relay position and the allocated time for such a resource allocation, and compare those in CR with those in multi-hop relaying, which does not have the asymmetric property, in order to evaluate the impact.

\subsection{Problem Definition}

This resource allocation problem can be formulated as follows:

$$
\begin{aligned}
\max _{x_{\mathrm{R}}, y_{\mathrm{R}}, \tau_{\mathrm{S}_{1}}} & \tau_{\mathrm{S}_{1}} \mathrm{E}\left[f_{\mathrm{S}_{1} \rightarrow \mathrm{S}_{2}}\right]+\left(1-\tau_{\mathrm{S}_{1}}\right) \mathrm{E}\left[f_{\mathrm{S}_{2} \rightarrow \mathrm{S}_{1}}\right] \\
\text { subject to } & \tau_{\mathrm{S}_{1}} \mathrm{E}\left[f_{\mathrm{S}_{1} \rightarrow \mathrm{S}_{2}}\right]:\left(1-\tau_{\mathrm{S}_{1}}\right) \mathrm{E}\left[f_{\mathrm{S}_{2} \rightarrow \mathrm{S}_{1}}\right] \\
& =T_{\mathrm{S}_{1}}: T_{\mathrm{S}_{2}}=1: k,
\end{aligned}
$$

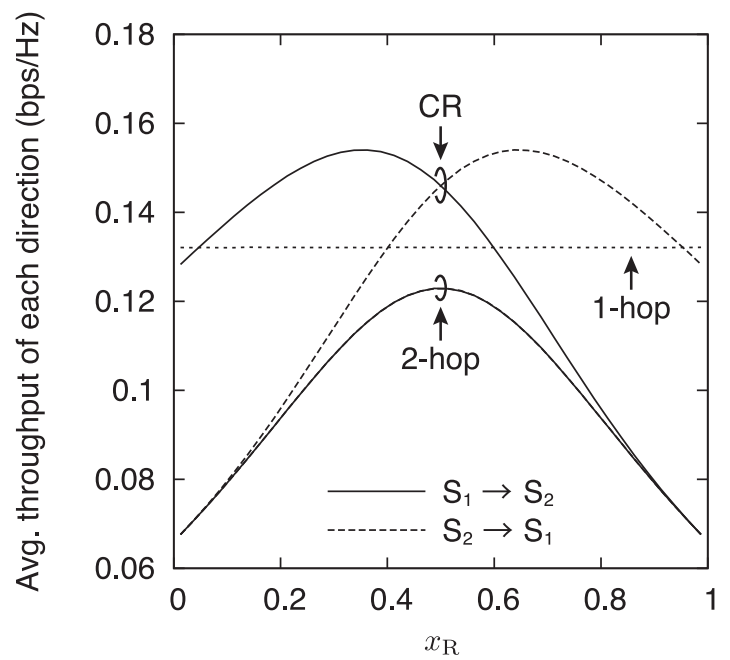

(a) Average throughput of each direction when $\mathrm{R}$ is assumed to be on the straight line segment from $\mathrm{S}_{1}$ to $\mathrm{S}_{2}$, i.e., $y_{\mathrm{R}}=0$.

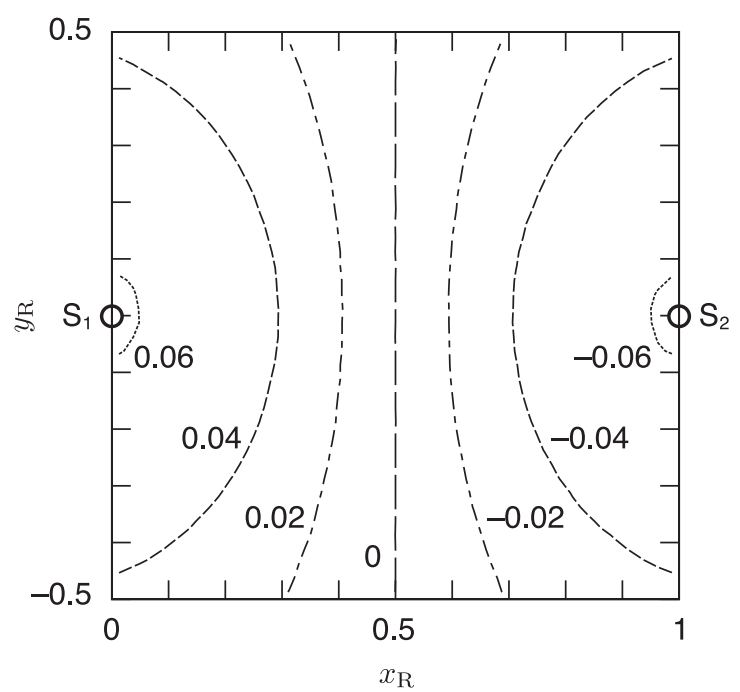

(b) Difference in the average throughput for each direction $\mathrm{E}\left[f_{\mathrm{S}_{1} \rightarrow \mathrm{S}_{2}}^{\mathrm{CR}}\right]-\mathrm{E}\left[f_{\mathrm{S}_{2} \rightarrow \mathrm{S}_{1}}^{\mathrm{CR}}\right]$.

Fig. 3 Asymmetric property of CR. The average end-to-end SNR is set to $-10 \mathrm{~dB}$. 
where $\tau_{\mathrm{S}_{1}}$ denotes the allocated time for the communication from $\mathrm{S}_{1}$ to $\mathrm{S}_{2}$ per unit time, $T_{X}$ denotes the offered traffic at station $X$, and $k$ denotes a given traffic ratio $T_{\mathrm{S}_{2}} / T_{\mathrm{S}_{1}}$. Here, $f_{\mathrm{S}_{1} \rightarrow \mathrm{S}_{2}} \in\left\{f_{\mathrm{S}_{1} \rightarrow \mathrm{S}_{2}}^{\mathrm{CR}}, f_{\mathrm{S}_{1} \rightarrow \mathrm{S}_{2}}^{2-\text { hop }}\right\}, f_{\mathrm{S}_{2} \rightarrow \mathrm{S}_{1}} \in\left\{f_{\mathrm{S}_{2} \rightarrow \mathrm{S}_{1}}^{\mathrm{CR}}, f_{\mathrm{S}_{2} \rightarrow \mathrm{S}_{1}}^{2-\text { hop }}\right\}$. Note that the average throughputs of the communication from $S_{1}$ to $S_{2}$ and from $S_{2}$ to $S_{1}$ will be $\tau_{S_{1}} E\left[f_{S_{1} \rightarrow S_{2}}\right]$ and $\left(1-\tau_{\mathrm{S}_{1}}\right) \mathrm{E}\left[f_{\mathrm{S}_{2} \rightarrow \mathrm{S}_{1}}\right]$, respectively. Using this problem definition, we evaluate the differences between the two relaying schemes with respect to the optimal relay position and the total throughput of bi-directional communication.

The communication between $S_{1}$ and $S_{2}$ is assumed to select one relay station from candidates of relay station. Here we assume a lot of relay candidates and discuss the relay position that realizes the largest throughput. Since the throughput is dependent on a given traffic radio, the optimal relay position is also dependent on the given traffic ratio.

\subsection{Evaluation}

Before evaluating the optimal relay position and total throughput, we address the difference in rate region between the two relaying schemes.

Figure 4 shows the rate regions of two-hop relaying and $\mathrm{CR}$, where the relay station is located at $\mathrm{S}_{1} \mathrm{R} / \mathrm{S}_{1} \mathrm{~S}_{2}=$ $0.2 \sim 0.8$. In the figure, each line represents the rate region at which the relay station is fixed and the allocated time is changed, and each point on the line is a vector of achievable rates that can be maintained in each direction simultaneously. The parameters used in the evaluation are as described in Sect. 3. The slope of each bold dashed line represents a traffic ratio. The optimal point can be calculated as the intersection of the line in the rate region with the bold line, where the total throughput is maximized under the constraint of achieving a given traffic ratio.

In $\mathrm{CR}$, the line that intersects the bold line changes depending on the slope of the bold line, whereas in two-hop relaying, the line that intersects the bold line does not change. In other words, in CR, the optimal relay position changes depending on the traffic ratio, whereas in two-hop relaying, the optimal relay position does not change. Since two-hop relaying has no directional throughput difference, all of the lines are parallel. Therefore, the optimal relay position is independent of the traffic ratio, and only the allocated time changes. On the other hand, since the average throughput in CR differs for each direction, the slopes of the lines in the region are different, and the optimal relay position changes depending on the slope of the bold line, i.e., the traffic ratio.

Figure 5 shows the optimal relay position to maximize the total throughput of bi-directional communication under the constraint of achieving a given traffic ratio. In $\mathrm{CR}$, the optimal relay position changes depending on the traffic ratio, whereas in two-hop relaying, the optimal relay position does not change. In two-hop relaying, the relay position does not affect the ratio of each average throughput, but rather the total throughput of bi-directional communication because there is no difference in average throughput between directions. Therefore, in order to maximize the total through-

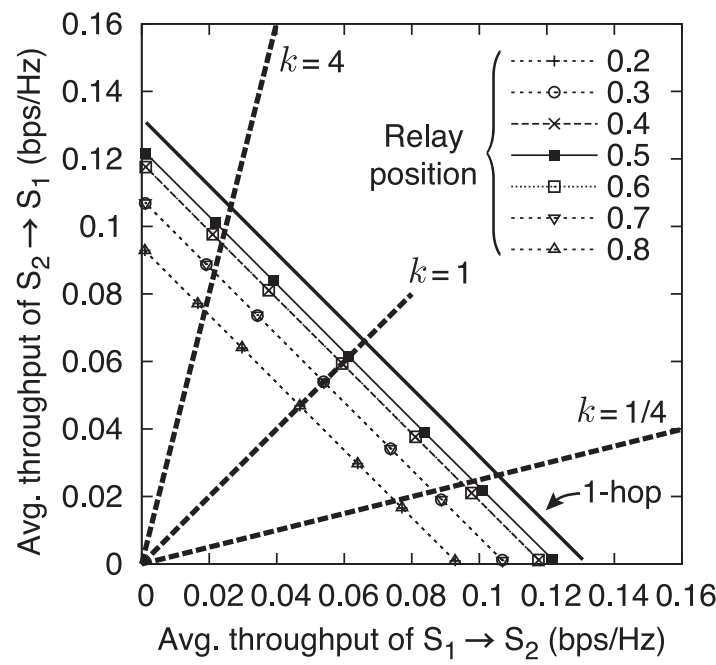

(a) Rate region of two-hop relaying.

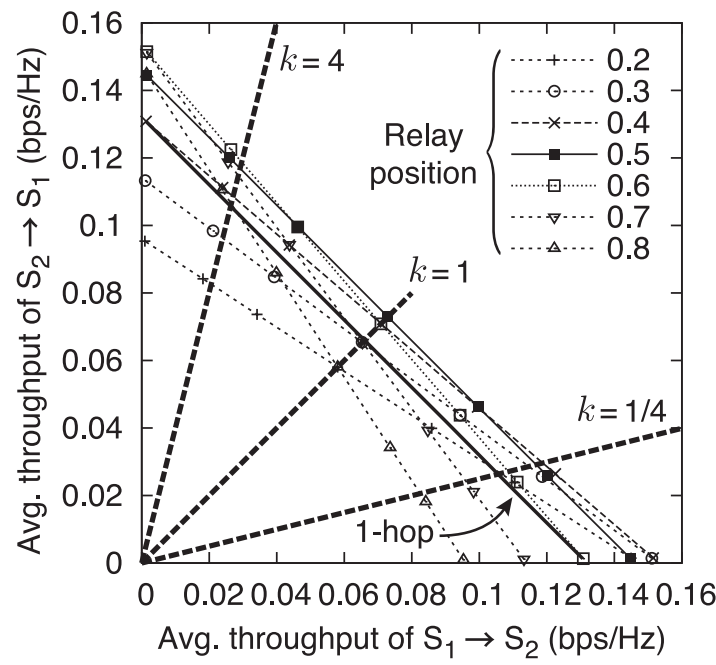

(b) Rate region of cooperative relaying.

Fig. 4 Rate regions. Each line represents the rate region at which the relay station is fixed and the allocated time is changed. Each point on the line is a vector of achievable rates that can be maintained in each direction simultaneously. The slope of each bold dashed line represents a traffic ratio.

put in two-hop relaying, the relay station should always be located halfway between end stations. On the other hand, the average throughput in CR differs for each direction, and, as a result, the optimal relay position has an effect on not only the total throughput but also the ratio of each average throughput. Therefore, the relay station should be selected according to the ratio $k$.

The optimal relay position is also dependent on the end-to-end SNR. Along with an increase in the end-to-end SNR, the optimal relay position tends to be close to the center between $S_{1}$ and $S_{2}$ because the average throughput is mainly determined by the end-to-end SNR and thus the difference in average throughput for each direction is small.

Figure 6 shows the optimal total throughput of bidirectional communication. In order to evaluate the impact of the asymmetric property caused by a shift in the relay po- 


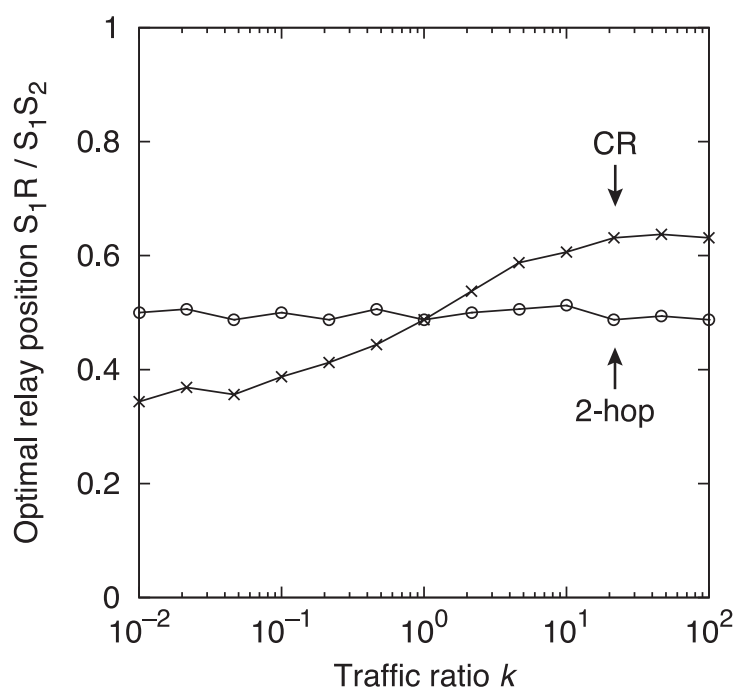

Fig.5 Optimal relay position to maximize the total throughput of bi-directional communication.

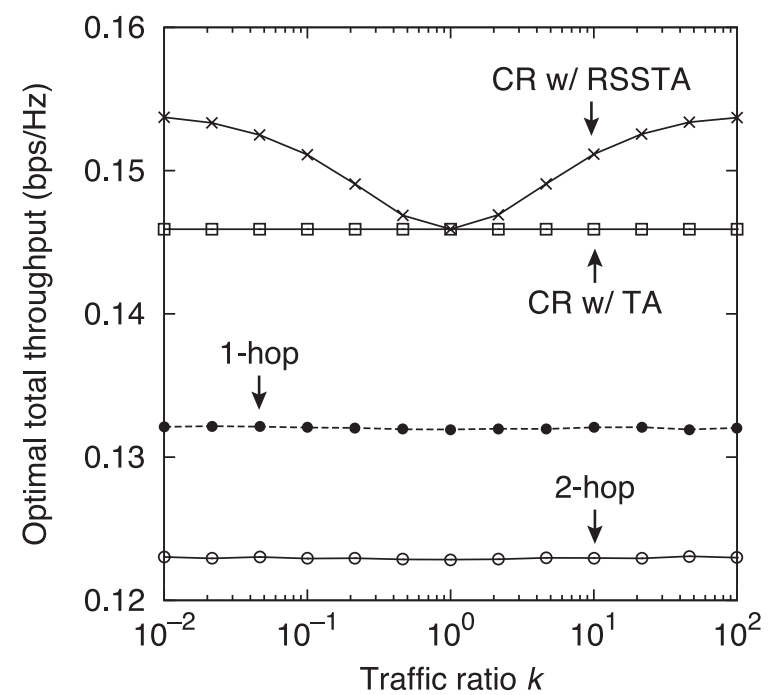

Fig. 6 Optimal total throughput of bi-directional communication to maximize the total throughput. In these figures, RSSTA denotes relay station selection and time allocation, and TA denotes time allocation.

sition on the total throughput, we assume the CR with only time allocation (TA), where the relay station is assumed to be located halfway between end stations. In two-hop relaying, the total throughput of bi-directional communication does not change because the optimal relay position is unchanged, as shown in Fig. 5, and time allocation has no effect on the total throughput. In CR with only time allocation, the result is the same as in the case of two-hop relaying because of the assumption that the relay position is fixed. In contrast, in CR with both relay station selection and time allocation (RSSTA), the total throughput changes depending on the ratio due to the shift in the relay position.

Figure 7 shows the optimal allocated time for the communication from $S_{1}$ to $S_{2}$ per unit time. In single-hop transmission or two-hop relaying, there is no difference between

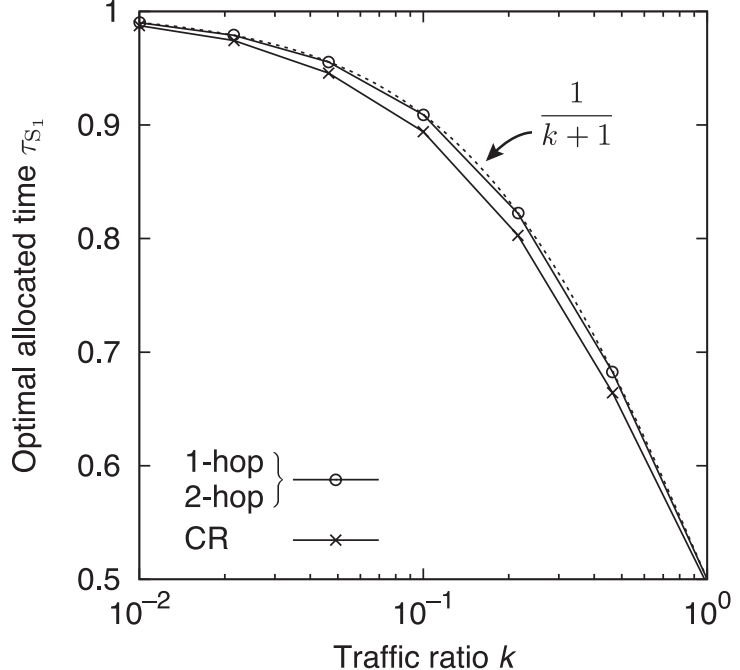

Fig. 7 Optimal allocated time for the communication from $S_{1}$ to $S_{2}$ per unit time.

$\mathrm{E}\left[f_{\mathrm{S}_{1} \rightarrow \mathrm{S}_{2}}\right]$ and $\mathrm{E}\left[f_{\mathrm{S}_{2} \rightarrow \mathrm{S}_{1}}\right]$. Thus the optimal allocated time ratio $\tau_{\mathrm{S}_{1}}$ should be set as $1 /(k+1)$, which is derived from the relation $\tau_{\mathrm{S}_{1}}:\left(1-\tau_{\mathrm{S}_{1}}\right)=1: k$, and it is confirmed by Fig. 7 .

\section{Opportunistic Transmission Direction Switching}

In this section, we discuss opportunistic transmission direction switching schemes using the asymmetric property of $\mathrm{CR}$, especially the difference in the instantaneous communication quality for each direction. When a traffic ratio is assumed, the total throughput of bi-directional communication must be enhanced under the constraint that the assumed traffic ratio is achieved. In this section, in order to satisfy this requirement, we propose an opportunistic transmission direction switching scheme: weighted proportional fair scheduling (WPFS), and compare two parameters, the total throughput and the throughput ratio, which is defined as the ratio of each average throughput, in the WPFS with those in the maximum total throughput (MTT) and in the weighted round robin (WRR). Here, we define the throughput ratio as the ratio of the average throughputs for each direction derived as a result of the switching.

\subsection{Total Throughput of Switching Schemes}

\subsubsection{Weighted Round Robin}

In the TDD system, the end stations have to switch the direction in order to communicate in both directions. In the WRR, each end station statically switches on periodic bases $\frac{1}{1+k} T$ and $\frac{k}{1+k} T$, respectively, in order to achieve a given traffic ratio. Then, the total throughput of bi-directional communication $f_{\mathrm{WRR}}$ at time $t$ can be expressed as 


$$
f_{\mathrm{WRR}}(t)= \begin{cases}f_{\mathrm{S}_{1} \rightarrow \mathrm{S}_{2}}(t) & n T \leq t<\left(n+\frac{1}{1+k}\right) T \\ f_{\mathrm{S}_{2} \rightarrow \mathrm{S}_{1}}(t) & \left(n+\frac{1}{1+k}\right) T \leq t<(n+1) T\end{cases}
$$

with any natural number $n$.

\subsubsection{Maximum Total Throughput}

The MTT is an opportunistic scheme to maximize the total throughput of bi-directional communication. In the MTT scheme, the end stations switch the direction when the direction with higher instantaneous throughput changes. The total throughput $f_{\text {MTT }}$ can be expressed as

$$
f_{\mathrm{MTT}}(t)=\max _{X} f_{X}(t)
$$

where $X \in\left\{\mathrm{S}_{1} \rightarrow \mathrm{S}_{2}, \mathrm{~S}_{2} \rightarrow \mathrm{S}_{1}\right\}$ denotes the transmission direction.

\subsubsection{Weighted Proportional Fair Scheduling}

In the WPFS, the end stations opportunistically switch the direction depending on three parameters: the instantaneous throughput, the average throughput, and the offered traffic. The WPFS was proposed in [13] in the context of downlink scheduling in a cellular network and provides weighted proportional fairness [14]. In the present study, we apply the WPFS to transmission direction switching in order to both enhance the total throughput and achieve a given traffic ratio. The total throughput $f_{\mathrm{WPFS}}$ can be expressed as

$$
f_{\mathrm{WPFS}}(t)=\max _{X} T_{X} \frac{\left(f_{X}(t)\right)^{a}}{W_{X}(t)},
$$

where $a$ is a parameter of the scheduler design, and $W_{X}(t)$ denotes the average throughput at time $t$. As $a$ becomes larger, the end stations communicate in the direction with higher instantaneous throughput. Here, $W_{X}(t)$ is averaged using an exponentially weighted lowpass filter parameterized by $\psi$. Assuming that, at time $t$, the direction $X^{\star}$ has the highest ratio, $W_{X}(t+1)$ is updated as

$$
W_{X}(t+1)= \begin{cases}(1-\psi) W_{X}(t)+\psi f_{X}(t) & X=X^{\star} \\ (1-\psi) W_{X}(t) & X \neq X^{\star}\end{cases}
$$

Note that the WRR and WPFS are traffic-aware switching schemes.

\subsection{Numerical Results}

Table 2 summarizes the parameters used in the evaluation. We assume path loss with path loss exponent $\alpha$. For the sake of simplicity, the effect of shadowing is not considered. The relay station is assumed to be located on a straight-line segment from $S_{1}$ to $S_{2}$.
Table 2 Parameters used in the evaluation.

\begin{tabular}{c|c}
\hline Parameters & Values \\
\hline Path loss exponent $\alpha$ & 2 \\
Channel model & Rayleigh fading \\
Area environment & Noise-limited \\
\hline
\end{tabular}

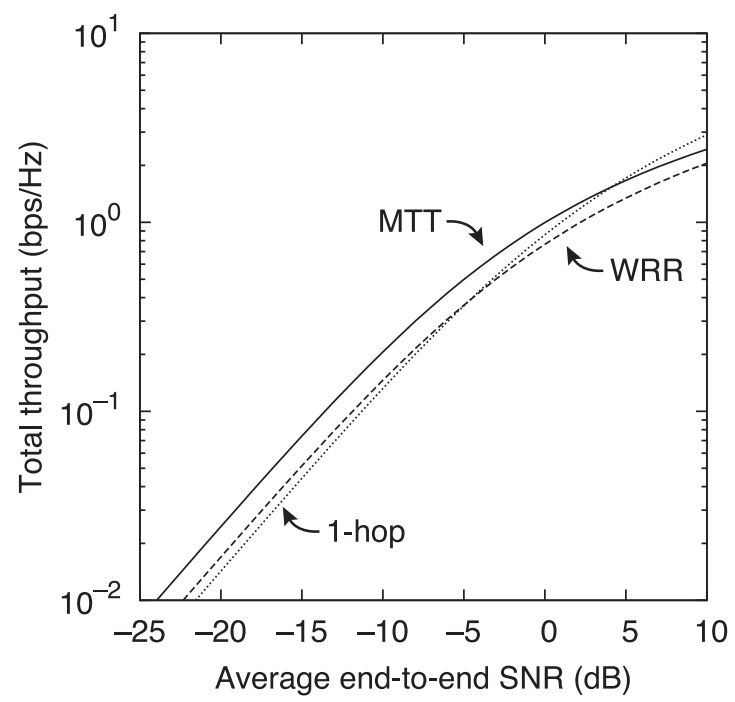

Fig. 8 Total throughput in one transmission direction. Note that the average throughput in each transmission direction are equal because the relay station is located halfway between the end stations.

\subsubsection{Trade-Off between Total Throughput and Through- put Ratio}

First, in order to characterize the MTT and WRR, we provide a preliminary evaluation of the average throughput in the MTT and WRR. In this evaluation, a system in which each end station is required to send the same amount of data $k=1$ is assumed, and the relay station is assumed to be located halfway between the end stations.

Figure 8 shows the total throughput versus the end-toend SNR. Note that the MTT always outperforms the WRR because, in the MTT, the end stations always communicate in the direction with higher instantaneous throughput. Here, the relay station is assumed to be located halfway between the end stations, i.e., the average channel gains of the R- $S_{1}$ link and the $\mathrm{R}-\mathrm{S}_{2}$ link are equal. However, the instantaneous channel gains are different, which leads to the difference in the instantaneous throughput for each direction.

Figure 9 shows the transmission time ratio of each direction and the average throughput of each direction, when the average channel gains of the R-S $S_{1}$ link and the $\mathrm{R}-\mathrm{S}_{2}$ link are different and the average end-to-end SNR is set to $-10 \mathrm{~dB}$. The transmission time ratio represents the percentage of the actual transmission duration in each direction. We observe that the transmission time ratio and the average throughput in the MTT change depending on the relay position.

In the MTT, when the relay station is located near $S_{1}$, 


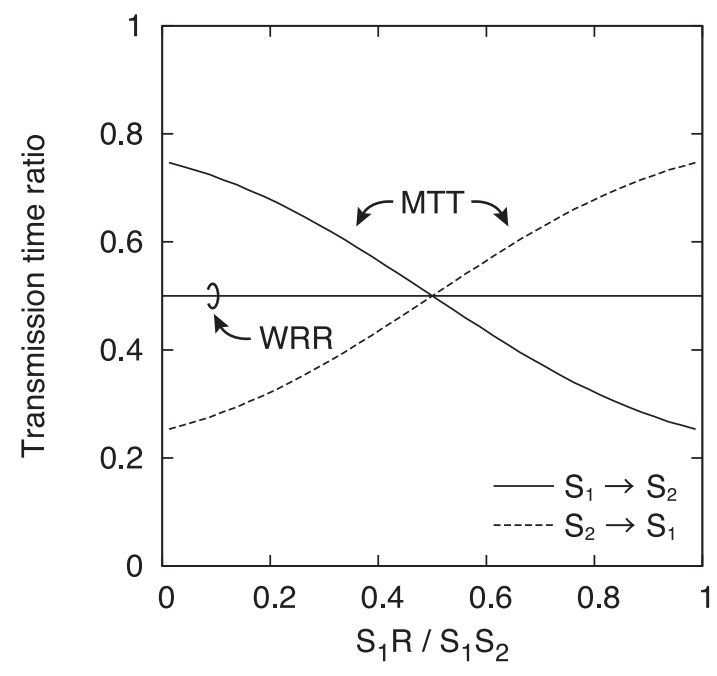

(a) Transmission time ratio of each transmission direction vs. the relay position.

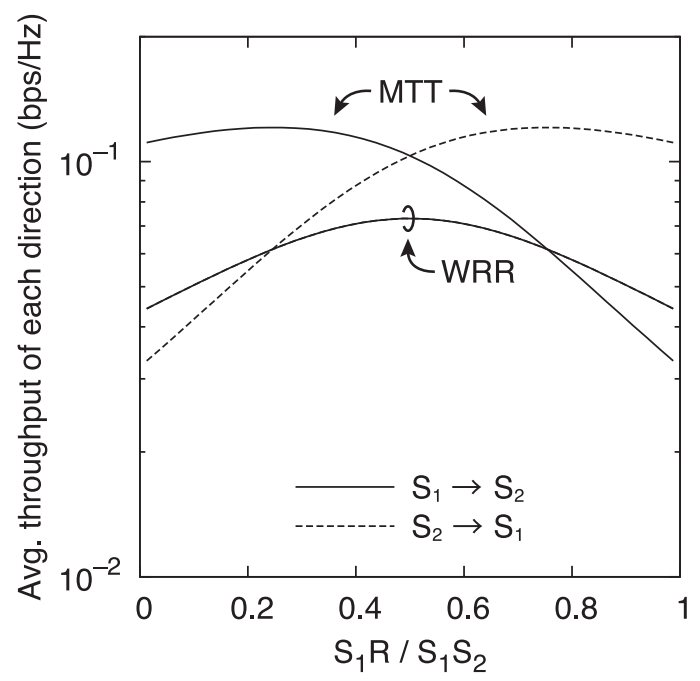

(b) Throughput of each transmission direction vs. the relay position.

Fig. 9 Transmission time ratio and throughput of each direction in the MTT.

$\mathrm{S}_{2}$ transmits the signal for a shorter time than $\mathrm{S}_{1}$. This is because the instantaneous channel gain of the R-S link is almost always higher than that of the $\mathrm{R}-\mathrm{S}_{2}$ link, which is a result of the average channel gain of the $\mathrm{R}-\mathrm{S}_{1}$ link being higher than that of the R-S $\mathrm{S}_{2}$ link. In this case, there is a significant difference in throughput for each direction, whereas the WRR can achieve the necessary traffic ratio independently of the relay position.

In this way, there is a trade-off between the total throughput and the throughput ratio: the highest total throughput can be achieved in the MTT, and the throughput ratio closest to the traffic ratio can be achieved in the WRR. Therefore, an opportunistic scheme, which can achieve both high total throughput and a throughput ratio close to the traffic ratio, is required.

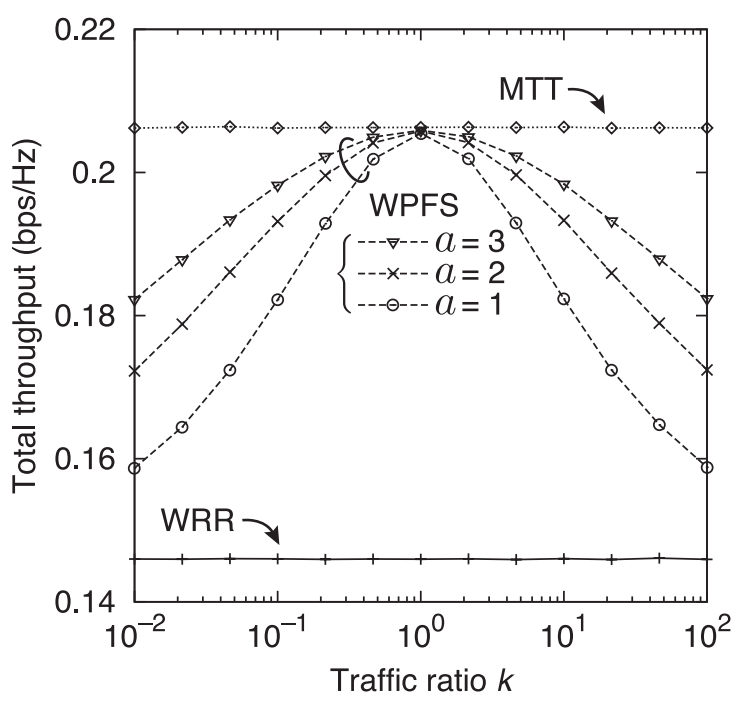

(a) Total throughput.

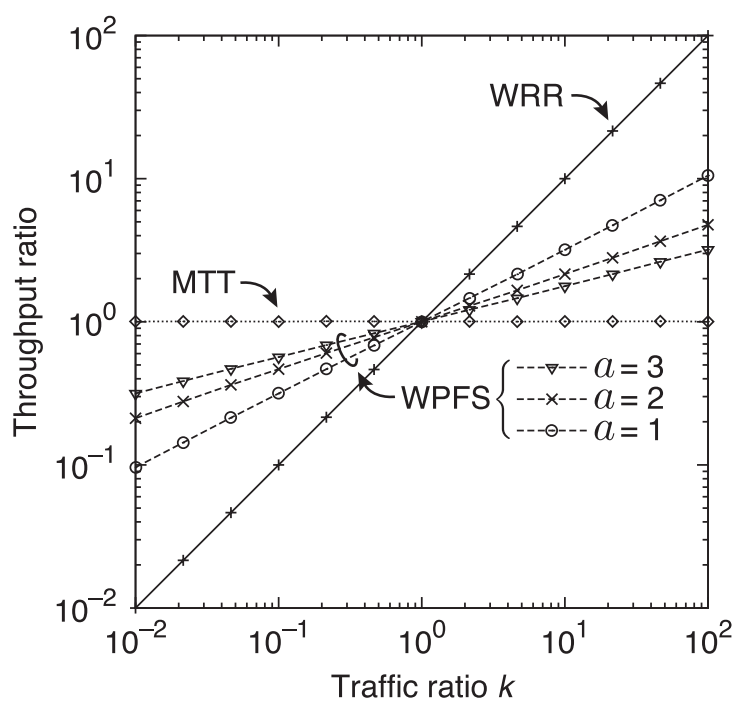

(b) Throughput ratio.

Fig. 10 Total throughput of bi-directional communication and throughput ratio of each transmission direction switching scheme. The relay station is located at $S_{1} R / S_{1} S_{2}=1 / 2$.

\subsubsection{Evaluation of the WPFS}

Next, we apply the WPFS to transmission direction switching in order to achieve both high total throughput and a throughput ratio close to the traffic ratio. In the following evaluation, the relay station is assumed to be located at $S_{1} R / S_{1} S_{2}=1 / 2,1 / 8$. The average end-to-end SNR is set to $-10 \mathrm{~dB}$.

Figures 10 and 11 show the total throughput and throughput ratio. As mentioned previously, the highest total throughput is achieved in the MTT, and the throughput ratio closest to the traffic ratio is achieved in the WRR. In contrast, the WPFS provides higher total throughput than the WRR and a throughput ratio that is closer to the traffic ratio, 


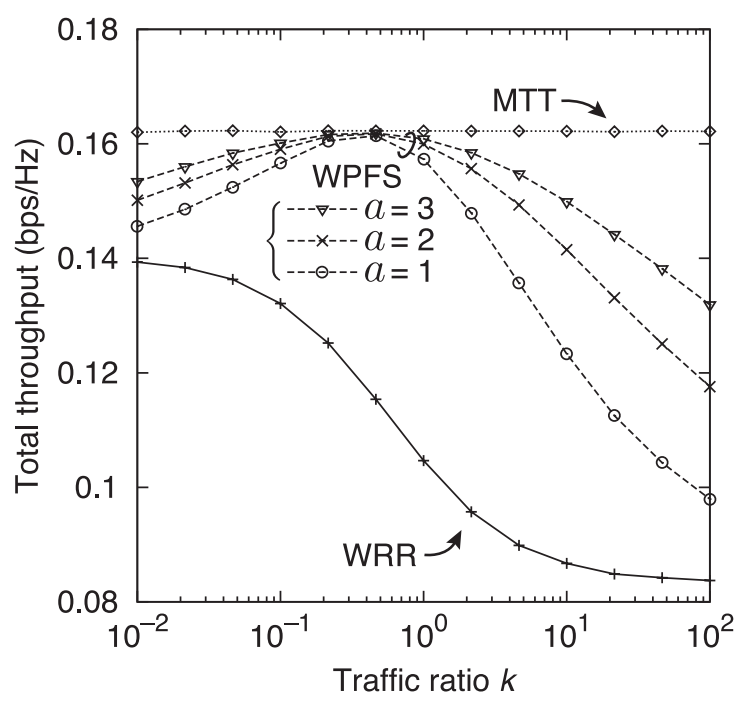

(a) Total throughput.

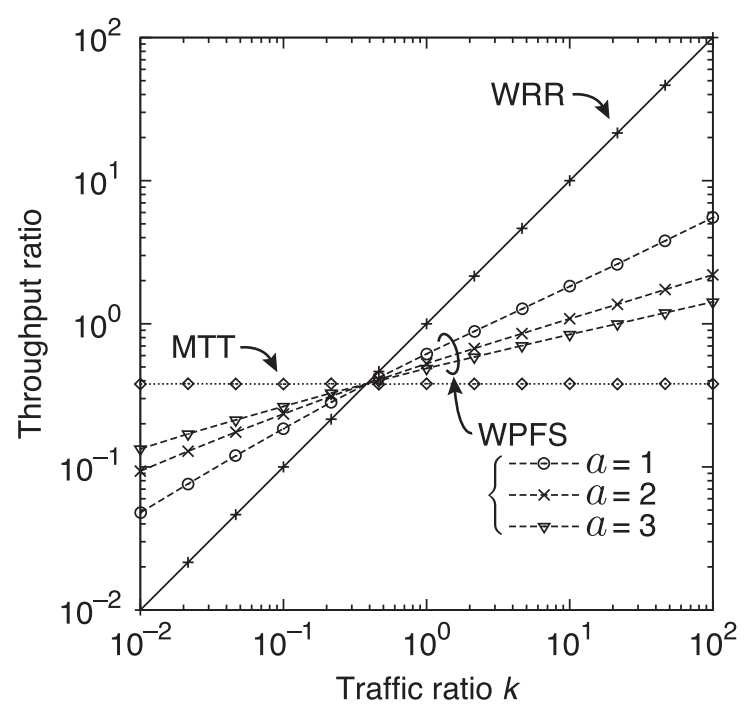

(b) Throughput ratio.

Fig. 11 Total throughput of bi-directional communication and throughput ratio of each transmission direction switching scheme. The relay station is located at $S_{1} R / S_{1} S_{2}=1 / 8$.

as compared to the MTT, where the value of $a$ can be used to tune the trade-off.

In both Figs. 10 and 11, especially when the traffic ratio is close to the throughput ratio of the MTT, the WPFS achieves better performance with respect to the two parameters. In the MTT, the end stations switch the direction depending on the instantaneous throughput of each direction. On the other hand, in the WPFS, the end stations switch the direction depending not only on the instantaneous throughput but also on the average throughput of each direction and the traffic ratio. When the traffic ratio is close to the throughput ratio of the MTT, which is derived as a result of the switching to maximize the total throughput, the WPFS can enhance the total throughput with a throughput ratio close to the traffic ratio.

\section{Conclusion}

We discussed the impact and use of the asymmetric property in bi-directional CR under asymmetric traffic conditions in the TDD system. First, we evaluated the average throughput of bi-directional CR. Numerical results revealed the asymmetric property of average throughput in CR.

Next, we discussed the impact of the asymmetric property on the optimal relay position and time allocation. In order to evaluate this impact, we evaluated the differences between the two relaying schemes with respect to the optimal relay position and total throughput. Numerical results indicated that, in order to maximize the total throughput of bi-directional communication, the relay station should be selected depending on the offered traffic ratio in CR. In contrast, the optimal relay position does not necessarily depend on the ratio in two-hop relaying, i.e., the total throughput can be improved by using the asymmetric property of CR under asymmetric traffic conditions.

Finally, we exploited the asymmetric property to enhance the total throughput of bi-directional communication and applied WPFS, which was originally proposed in the context of downlink scheduling in a cellular network, to transmission direction switching. Numerical results revealed that the WPFS provides high total throughput and a throughput ratio close to the offered traffic ratio.

\section{Acknowledgments}

The present study is supported in part by a Grant-in-Aid for Scientific Research (A) (no. 20246067) from the Japan Society for the Promotion of Science (JSPS).

\section{References}

[1] R. Nabar, H. Bölcskei, and F. Kneübuhler, "Fading relay channels: Performance limits and space-time signal design,” IEEE J. Sel. Areas Commun., vol.22, no.6, pp.1099-1109, Aug. 2004.

[2] J. Laneman, D. Tse, and G. Wornell, "Cooperative diversity in wireless networks: Efficient protocols and outage behavior," IEEE Trans. Inf. Theory, vol.50, no.12, pp.3062-3080, Dec. 2004.

[3] M. Haenggi, "Analysis and design of diversity schemes for ad hoc wireless networks," IEEE J. Sel. Areas Commun., vol.23, no.1, pp.19-27, Jan. 2005.

[4] K. Yamamoto, H. Maruyama, T. Shimizu, H. Murata, and S. Yoshida, "Spectral efficiency of fundamental cooperative relaying in interference-limited environments," IEICE Trans. Commun., vol.E91-B, no.8, pp.2674-2682, Aug. 2008.

[5] P. Larsson, N. Johansson, and K. Sunell, "Coded bi-directional relaying,” Proc. IEEE VTC 2006-Spring, vol.2, pp.851-855, May 2006.

[6] S. Fu, K. Lu, Y. Qian, and M. Varanasi, "Cooperative network coding for wireless ad-hoc networks," Proc. IEEE GLOBECOM'07, pp.812-816, Nov. 2007.

[7] B. Rankov and A. Wittneben, "Spectral efficient protocols for halfduplex fading relay channels," IEEE J. Sel. Areas Commun., vol.25, no.2, pp.379-389, Feb. 2007.

[8] Y. Han, S. Ting, C. Ho, and W. Chin, "High rate two-way amplifyand-forward half-duplex relaying with OSTBC," Proc. IEEE VTC 2008-Spring, pp.1550-2252, May 2008. 
[9] N. Hu, X. Zhong, M. Zhao, and J. Wang, "Regenerative strategy for fairness in bi-directional three-node relaying," IEICE Trans. Commun., vol.E91-B, no.8, pp.2736-2739, Aug. 2008.

[10] S.J. Kim, N. Devroye, P. Mitran, and V. Tarokh, "Comparison of bi-directional relaying protocols," IEEE Sarnoff Symposium 2008, pp.1-5, April 2008.

[11] C.H. Liu and F. Xue, "Network coding for two-way relaying: Rate region, sum rate and opportunistic scheduling," Proc. IEEE ICC'08, pp.1044-1049, May 2008.

[12] R. Nabar and H. Bölcskei, "Space-time signal design for fading relay channels," Proc. IEEE GLOBECOM’03, vol.4, pp.1952-1956, Dec. 2003.

[13] R. Agrawal, A. Bedekar, R. La, and V. Subramanian, "Class and channel condition based weighted proportionally fair scheduler," Teletraffic Engineering in the Internet Era, Proc. ITC 2001, vol.17, pp.553-565, Sept. 2001.

[14] F. Kelly, A. Maulloo, and D. Tan, "Rate control in communication networks: Shadow prices, proportional fairness and stability," Journal of the Operations Research Society, vol.49, no.3, pp.237-252, March 1998.

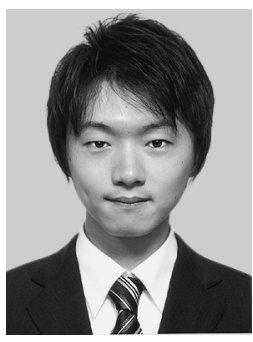

Takaaki Saeki received the B.E. degree in electrical and electronic engineering from Kyoto University in 2008, and the M.E. degree in informatics from Kyoto University in 2010. He is currently working in Sony Corporation, Japan. His research interests include cooperative relaying systems.

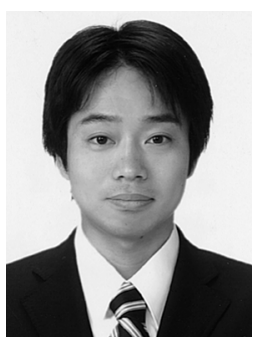

Koji Yamamoto received the B.E. degree in electrical and electronic engineering from Kyoto University in 2002, and the M.E. and Ph.D. degrees in informatics from Kyoto University in 2004 and 2005, respectively. Since 2005, he has been an assistant professor of the Graduate School of Informatics, Kyoto University. From 2008 to 2009 , he was a visiting researcher at Wireless@KTH, Royal Institute of Technology $(\mathrm{KTH})$ in Sweden. His research interests include game theory, spectrum sharing, and cooperative multi-hop networks. He received the PIMRC 2004 Best Student Paper Award in 2004, the Ericsson Young Scientist Award in 2006, and the Young Researcher's Award from the IEICE of Japan in 2008. He is a member of the IEEE.

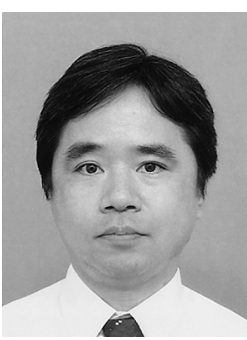

Hidekazu Murata received the B.E., M.E., and Ph.D. degrees in electronic engineering from Kyoto University, Kyoto, Japan, in 1991, 1993, and 2000, respectively. In 1993, he joined the Faculty of Engineering, Kyoto University. From 2002 to 2006, he was an Associate Professor of Tokyo Institute of Technology. He has been at Kyoto University since October 2006 and is currently an Associate Professor of Department of Communications and Computer Engineering, Graduate School of Informatics. His major research interests include signal processing and its hardware implementation, with particular application to cooperative wireless networks with cognitive radio capabilities. He received the Young Researcher's Award from the IEICE of Japan in 1997, the Ericsson Young Scientist Award in 2000, and the Young Scientists' Prize of the Commendation for Science and Technology by the Minister of Education, Culture, Sports, Science and Technology in 2006. He is a member of the IEEE and SITA.

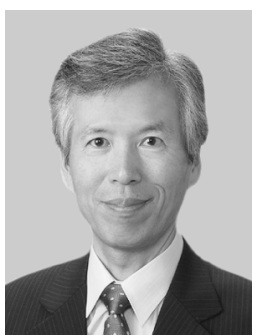

Susumu Yoshida received the B.E., M.E. and $\mathrm{Ph} . \mathrm{D}$. degrees all in electrical engineering from Kyoto University, Kyoto, Japan in 1971, 1973 and 1978, respectively. Since 1973, he has been with the Faculty of Engineering, Kyoto University and currently he is a full professor of the Graduate School of Informatics, Kyoto University. During the last 30 years, he has been mainly engaged in the research of wireless personal communications. His current research interest includes highly spectrally efficient wireless transmission techniques and distributed controlled wireless networks. During 1990-1991, he was a visiting scholar at WINLAB, Rutgers University, U.S.A. and Carleton University in Ottawa, Canada. He served as a TPC Chair of IEEE VTC 2000-Spring, Tokyo. He was a guest editor of IEEE J-SAC on Wireless Local Communications published in April and May 1996. He received the IEICE Achievement Award and Ericsson Telecommunication Award in 1993 and 2007, respectively. 